\title{
7. Accessibility of data visualizations: An overview of European statistics institutes
}

\author{
Mikael Snaprud and Andrea Velazquez
}

\begin{abstract}
Access to public data is important for people to stay informed. Access to visualizations of national statistics can be essential in order to take part in political discussions and so to shape a democratic society. In this chapter we investigate accessibility for people with disabilities to data visualizations from a selection of European National Statistics Institutes (NSIs). We outline related practices and approaches to accessibility improvements and propose a way to evaluate and compare accessibility aspects of data visualizations. The findings indicate that in contrast to the recently harmonized European legal requirements, the degree to which the data visualizations meet the requirements, and the approaches to meet them, are very different among the NSIs across Europe.
\end{abstract}

Keywords: Data visualization; Accessibility; Web Accessibility Directive; National Statistics Institutes; NSI.

\section{Introduction}

Data visualizations can inform citizens about political topics, and access to them for all citizens, regardless of ability and related technology use, is essential for democratic processes. The United Nations Convention on the Rights of Persons with Disabilities requires that appropriate measures are taken to ensure access for persons with disabilities, on equal basis with others, to information and communication technologies, including the internet. The European Web Accessibility Directive (WAD), transposed into

Engebretsen, M. and H. Kennedy (eds.), Data Visualization in Society. Amsterdam: Amsterdam University Press, 2020 DOI 10.5117/9789463722902_CHO7 
national law for all the EU member states from September 2018, makes web accessibility a legal obligation. It requires that public sector bodies provide accessibility statements, including a list of content that is not accessible, the reasons for the inaccessibility and accessible alternatives to it, and a feedback mechanism for users to report accessibility problems on all of their websites, including in relation to data visualizations. National Statistics Institutes (NSIs) are a key source of such visualizations. Therefore, in this chapter, we focus on the accessibility of data visualizations produced and provided by European NSIs. We present results from our evaluation of the accessibility of data visualizations on NSI websites and from research with NSIs regarding their preparations to conform with the Directive.

In order to be accessible, the data visualizations (DVs), like all other web content, need to be perceivable, operable, understandable, and robust. For this chapter we leave out understandable, as an evaluation of such would require many more resources than we had available for our study. However, we add findability, the ease by which a piece of information on a website can be found (Jacob \& Loehrlein, 2009; Wikipedia, Findability, 2018), since it can have an impact on the ability of citizens to participate in democratic discourse.

Perceivability and operability of DVs are related to general website accessibility issues. For example, menus that cannot be used via keyboard navigation or input fields that are not properly labelled can cause problems for web users with disabilities. We used Tim Berners-Lee's 5 -star scheme, described below, to assess the robustness of data formats (5-star data, 2015).

We used an automated accessibility checker tool, WTKollen, to test the accessibility of the websites of 44 out of 59 European NSIs (WTKollen, European NSI sites, 2018), the results from which are presented as scores. ${ }^{1}$ The results presented below therefore refer only to the 44 websites we tested, and not to others which, for various reasons, it was not possible to test prior to publication of this chapter. We also carried out expert testing of data visualizations found on these websites, and conducted email surveys and semi-structured interviews with appropriate staff within the NSIs.

A limitation of our work is that we did not carry out user testing of the websites and DVs with web users with disabilities. This is widely deemed to be the most appropriate way of evaluating the accessibility of websites (e.g. Coyne \& Nielsen, 2001), but it is resource-intensive, especially in the case of EU-wide research such as ours. Automated checker tools, like accessibility measures more generally, also tend to privilege the needs of people with certain disabilities, such as visual impairment, and ignore the needs of 
others, such as intellectual disabilities (Kennedy, Thomas, \& Evans, 2010). It is also the case that some tests cannot be automated-for example, automatic image processing may not be able to distinguish a cat from a dog in a blurry picture to determine whether an alternative text for the picture is helpful or not-and this is another limitation of automated accessibility testing. Despite these limitations, we think that our research can provide valuable insights into the extent of the accessibility of DVs across European NSIs.

\section{Data collection}

\section{Automated accessibility testing}

The European Web Accessibility Directive is based on the Web Content Accessibility Guidelines (WCAG) from the World Wide Web Consortium $\left(\mathrm{W}_{3} \mathrm{C}\right)$. The guidelines are intended to cover any online content, including DVs, for people with disabilities, such as visual, auditory, physical, speech, cognitive, language, learning, and neurological disabilities. The WCAG 2.0 ( $\left.\mathrm{W}_{3} \mathrm{C}, 2008\right)$ was replaced by WCAG 2.1 in June 2018 (W3C, 2018). ${ }^{2}$ Following these guidelines will often make web content more accessible as well as serving other purposes. Proper use of alternative text descriptions on images, for example, can enable search engines to provide accurate search results. To guide the testing process, the $\mathrm{W}_{3} \mathrm{C}$ published the WCAG Evaluation Method 'WCAG-EM' in 2014 ( $\mathrm{W}_{3} \mathrm{C}$, 2014). This methodology offers guidance on the expertise required to test web accessibility, how to select webpages from a website, and how to report the findings. WCAG2.0 and WCAG-EM are therefore the basis for a range of web accessibility testing methods, tools, and legislation in Europe.

We carried out website accessibility evaluations with the WTKollen checker tool (WTKollen Page checker, 2018), which is based on WCAG 2.1 and WCAGEM 1.o. Whereas the WCAG-EM guidelines indicate what to look for to design the accessibility tests, they do not specify exactly how to implement tests. The applied tests are listed online (GitHub, 2018) — not all of them are equally relevant to all people with disabilities. For example, colour contrast can be important for a person with visual impairment while irrelevant for a blind user. Hence different user groups would assign different weights to the same test. For the score calculation, we needed to have one weight only for each test. Therefore, we decided to let all tests have the same impact on the score.

2 To better meet the needs for three major groups: users with cognitive or learning disabilities, users with low vision, and users with disabilities on mobile devices. 
To select pages from websites, we used a crawler, to find up to 6,00o pages. A random sample of 600 of these pages was used to represent the site to be tested. The score for a webpage was computed as the ratio of passed tests to applicable tests for each success criteria where associated tests applied. Similarly, the score for a website was aggregated over the test results for all the success criteria.

\section{Manual test procedure}

We uses a DV grouping proposed by Kirk (2012, p. 76) for the analysis of the DVs: Exploratory visualizations which aim to allow readers to discover features by interrogating the data themselves;

Explanatory visualizations which aim to convey specific information to readers, based on a predefined narrative;

Exhibitory visualizations which are also based on data, but contain an artistic element.

Further, we also grouped the DVs by ways of interacting with them:

Static visualizations, such as a PNG image;

Dynamic visualizations which move, but without users activating them; Navigable visualizations, which change based on user interaction;

Configurable visualizations, which enable users to select graph types or numbers, to move levels, or to select variables.

Our evaluation proceeded according to the following steps:

1. Locate the selected DV through a search on Google and local search, record rank (automatic accessibility check of the website as indication for how easy it is to navigate is done earlier)

2. Examine data presentation and downloadable data formats (i.e. how the data are provided, discussed below)

3. Group DV (according to Kirk's groupings discussed above)

4. Group DV (according to mode of interaction commented above)

5. Carry out manual accessibility tests for keyboard navigation, zoom, and textual description of the image (discussed below)

6. Look for accessibility feedback option (i.e. verify if there is an accessibility feedback mechanism on the page)

7. Look for supplementary services that may help the user understand the DV:

7.1. FAQ: Is there an FAQ available from the page? What kind of FAQ (general info, specific content)?

7.2. Languages: Is there support for multiple languages?

7.3. Glossary: Is there a glossary to explain terms used in the statistics on the page? 
The outcomes of the tests were recorded in a spreadsheet together with screenshots and URLs so that a test can be repeated if needed and for accountability purposes.

An important accessibility feature for people with motor impairments is the ability to navigate by using the keyboard or other input device instead of a mouse. If the page with the DV is designed to enable this, then users can reach all elements on the page and navigate forward and backward through the elements in the browser window with the tab and shift keys. Keyboard navigability can also allow users to select data and configure DVs.

The zoom feature is essential to magnify both text and images for people with visual impairments. Text zoom should render the text so that there is no need to scroll sideways. To test zoom features, it is necessary to explore whether the page has an option to enlarge the content or not. We evaluated the ability of the page to present the screen content enlarged.

There are two ways to provide text alternatives to images on webpages, thus making them accessible to people with visual disabilities: a short alternative text (alt-text) and a longer description: longdesc. The purpose of the longdesc is to provide more elaborate information when a short alt-text does not adequately convey the function or information provided in a non-text element on a webpage $\left(\mathrm{W}_{3} \mathrm{C}, 2016\right)$. Our test recorded whether the longdesc was used for complex DVs.

In many cases the data behind a DV are provided for download from the NSI site. Data formats have a strong impact on whether users can access and reuse the data. If a person is not able to use the DV, then a reusable data format can be more accessible and thus enable users to understand the data. Reusable data formats are also in line with the intention of the European Public Service Information directive, 'PSI' (EUR-Lex, 2005). The 5 -star scheme proposed by Tim Berners-Lee is a practical way of evaluating the extent to which a given dataset can be reused (5-star data, 2015).

1-Star: the data are open; however, they are locked-up in a document making it hard to get them out of the document, e.g. in a PDF or JPEG. 2 -Star: the data are accessible on the web in a structured way; however, they are still locked-up in a document depending on proprietary software, such as Microsoft Excel.

3-Star: the data are available on the web and can be manipulated in any way, without the need to own any proprietary software package, e.g. in CSV format.

4-Star: as above, and the data items have a URL and can be shared on the web, for example via links. 
5-Star: as above, and it is also possible to link data to other data to provide context, to discover more related data while consuming the data, thus benefiting from the network effect, e.g. through a link to a Wikipedia article.

More stars means more reusable and also to some extent more accessible. For example, a screen reader, used by blind users, will not be able to read data in a JPEG image. In addition, if access to data is only possible with proprietary software, then users without the software in question will be unable to access it. The context provided in the $4^{-}$and $5^{\text {-star levels }}$ does not really matter for accessibility, but is helpful for automated assessment of what the data are about. For our test, we detected if the data could be downloaded, and recorded the data format mapped to this 5 -star scheme.

Finally, for interactive DVs, we also recorded the following two properties. Comparability: number of variables that could be represented in the same graph.

Number of representations available: different kinds of charts available for representing the data, such as bar charts, linecharts, maps, pie charts.

\section{Web accessibility for the NSI websites}

The automated evaluation of website accessibility was carried out in the period from October 25 to November 13, 2018. The NSIs with the 12 highest scores are listed in Table 7.1. The highest score is awarded to the Irish NSI, followed by a group of 8 NSIs with a score of 99 . At the lower end of the list, we find the NSIs from Greenland (score 67), Cyprus (69), and Iceland (71). To view the full list and the details about the detected accessibility issues, visit the webpage (http://axe.checkers.wtkollen.se/en/benchmarking/ testrunresults/d235468e-65a4-43b2-8428-5908fo61fff9).

The above list was up-to-date at the time of publication. For the manual testing 14 NSIs were selected, based on high accessibility scores (in alphabetic order): Czech Republic (CZ), Denmark (DK), Germany (DE), Ireland (IE), Luxembourg (LU), The Netherlands (NL), Norway (NO), Poland (PL), Spain (ES), Sweden (SE), Switzerland (CH), and United Kingdom Visual ONS (UK-visual), and on a suggestion from Statistics Norway that they contain interesting DVs: Portugal (PT) and Slovenia (SI). 
Table 7.1 Overview of NSI websites and accessibility score from the WTKollen checker tool

\begin{tabular}{lllll}
\hline Rank & Score & Country & NSI short name & URL \\
\hline 1 & 100 & Ireland & Nisra UK & https://www.nisra.gov.uk/ \\
2 & 99 & Spain & INE ES & http://ine.es/ \\
3 & 99 & Sweden & SCB SE & http://www.scb.se/ \\
4 & 99 & Denmark & DST & http://www.dst.dk/ \\
5 & 99 & Germany & Statistikportal DE & http://www.statistik-portal.de/ \\
6 & 99 & Switzerland & BFS CH & http://www.bfs.admin.ch/ \\
7 & 99 & Norway & SSB NO & http://www.ssb.no/ \\
8 & 99 & Luxembourg & Statistiques & http://www.statistiques.public.lu/ \\
9 & 98 & United Kingdom & ONS UK & https://www.ons.gov.uk/ \\
10 & 98 & Czech Republic & CZSO CZ & https://www.czso.cz/ \\
11 & 98 & Poland & Stat PL & http://stat.gov.pl/ \\
12 & 97 & The Netherlands & CBS NL & https://www.cbs.nl/ \\
\hline
\end{tabular}

\section{DV findability on the NSI websites}

We searched for statistics about national population as a case to obtain an indicator of the DV findability on the NSIs websites. Population is well covered across all NSIs and it also seems to be a popular search topic. In a first attempt we used Google to search for 'population' and the name of the NSI. For Norway the search phrase was then 'population SSB'. For a corresponding search for each of the selected NSIs, all but one NSI appeared as the first item in the search results list. For thirteen out of fourteen NSI sites the local search returned the relevant page in rank one. Both the Google analytics data from Statistics Norway and the experience from Eurostat indicate that it is more common to search just for 'population' without any NSI portal name. For some searches the first result in Google is data from the World Bank. For Norway these data originate from Statistics Norway NSI.

A DV included in the Google search results list (Google public data, 2018), such as the one we found from SSB in Norway, can be convenient for the user, who may not need to look any further for the requested statistics. The DV we found had colour contrast issues, but otherwise it was quite accessible, using SVG graphics and offering the ability to present the data in several languages.

Our initial approach to test findability was to use similar English search phrases to find population DVs across all NSIs (e.g. 'population SSB' for Norway). In the course of the study we noted that the content on the NSI websites is 
mostly prepared for the national audience, to be searched in a national language. Therefore, a search in English across different NSI may produce results that are not relevant for the targeted national users. To refine this result we could do a new search for population in the local language. We also note that the search engine result can depend on who is doing the search and from where.

\section{Data presentation analysis}

The data presentation was assessed for the fourteen evaluated NSIs. The accessibility score is the result obtained in the period from September 2018 to November 2018. The accessibility scores of the population data presented are: Score of 95 to 99 , a few tests failed: DE

Score of 85 to 95 , some tests failed: ES, LU

Score of 70 to 85 , many tests failed: CZ, IE, NL, NO, PT, SE, UK-visual Score below 70, most tests failed: $\mathrm{CH}, \mathrm{DK}, \mathrm{PL}, \mathrm{SI}$

The data presented of twelve NSIs are configurable in tables in which it is possible to select the variables to show; only one is navigable (DE) allowing movement or arrangement of the data presented; and one static (UK-visual). For nine out the fourteen NSIs the keyboard navigation is enabled. Only four NSIs (CZ, ES, NL, NO) have the zoom feature; and in all but one (DE), the option to download the data is possible: CZ is 1-star; 10 NSIs are 3-star (that is, non-proprietary open format), NL 4-star and LU 5 -star.

\section{Exploratory/Interactive visualization analysis}

Twelve out of fourteen NSIs have interactive tools to graph the data which enable users to produce their own visual representations of the available data. CZ and UK-visual do not have interactive DVs. PT graphs need Adobe flash player which is not accessible for people with disabilities, and the DE tool is provided only in German and therefore not evaluated. Therefore, only ten DVs were manually tested.

Only four out of ten DVs could be checked automatically: DK, NL, NO, and ES, mostly because the generated graphics do not have an explicit link to enter into the checker tool. Single page applications will present the same URL independent of user configuration of the DV. The accessibility scores are:

ES scored 85 to 95 , some tests failed

NO and DK scored 70 to 85 , many tests failed

NL scored 65, most tests failed 
We note that all of the exploratory DVs tested have data to download, but only in a format not necessarily accessible and not suited for machine processing, mainly JPG and PNG format. Zooming is only supported by the DK, ES, NL, and NO examples and keyboard navigation is only supported in four out of ten cases (CH, ES, NL, PL). None of the DVs has longdesc enabled.

The DVs can be presented in different graphs depending on the nature of the data selected. For example, if the data selected do not include territories the map visualization is not a valid option. Some of the options available include bars, pie, lines, points, pyramid, and map. The number of graphs available is variable among NSIs, with the maximum fifteen $(\mathrm{CH})$ and the minimum two (ES and PL). All the tools tested can compare multiple variables. By their nature, all the DVs are configurable.

We can identify six different tools in use among the 10 NSIs assessed. The first one used in Norway, Ireland, Slovenia and Denmark. The second in Sweden and Switzerland. While Spain, the Netherlands, Luxembourg, and Poland all seem to use different tools.

\section{Explanatory visualization analysis}

A total of twelve NSIs were evaluated for explanatory DVs. For the Luxembourg and Slovenia NSIs we did not find any explanatory visualizations. The accessibility scores obtained are the following:

Score of 95 to 99, a few tests failed: CH, CZ, DE, DK, ES, NL, NO, PL, SE Score of 85 to 95 , some tests failed: IE, UK-visual

Score of 70 to 85 , many tests failed: PT

Eleven of the explanatory DVs tested are static and one is dynamic (NL). Only five out of the twelve have the option to download data:

1-star: CZ, IE, NO, PL

3-star: PT

Only two support keyboard navigation (NO, PT), five have the zoom ability (DE, ES, NL, NO, PL) and two support the longdesc (PT, UK-visual). The accessibility scores are higher for this category than for other DVs. This is because the content is typically simpler, mainly consisting of text and numbers. Even though the explanatory visualizations are simple, the option to download the data is not common. This makes the data harder to reuse. 


\section{Exhibitory visualization analysis}

Ten NSIs were assessed for their exhibitory visualizations; for the remaining four (DE, DK, LU, SE) we did not find any exhibitory DVs. Exhibitory DVs are produced like artistic posters, in different formats mainly in PDF formats and in PNG. The scores were also calculated with the PDF checker when necessary:

NO and PL: 95 to 99, a few tests failed

CZ, IE, NL, and UK-visual: 85 to 95 , some tests failed

SI PDF checker score: pass 6, fail 3

$\mathrm{CH}, \mathrm{ES}$, and PT PDF checker score: pass 5 , fail 2

Seven exhibitory DVs are static, two dynamic (CZ, NL), and one navigable (UK-visual). None has longdesc; and only NL support keyboard navigation. Five out of the nine exhibitory DVs have zoom ability, and seven have data to download, all are 1-star, making it hard to reuse the information.

\section{Services to support users to understand the DVs}

None of the fourteen evaluated NSIs has an accessibility feedback form, although most of them have a general feedback form to comment on the data or the page. One NSI page has a phone and email address for accessibility feedback and two NSIs have accessibility statements. By September 2020, all NSIs will need to have an accessibility feedback mechanism in place on their websites to conform to the Web Accessibility Directive.

FAQs are found on nine out of the fourteen NSIs. We found four FAQs containing general information about the page and five FAQs about specific content like consumer prices, wages, or summer prices. The option to select national language or English is provided by twelve out of fourteen NSIs. The UK-visual content is provided in English, and does not support any other language, possibly because this website is focused on DVs. The German NSI is only in German.

Glossary access to explain terms used on pages or in DVs is provided by nine out of fourteen NSIs. The glossary entries are found directly on the page, provided as references to an internal glossary or external ones like the ones from the OECD or from Eurostat's Statistics Explained (Eurostat, Statistics Explained, 2017).

\section{NSI practices relating to DV accessibility}

To supplement the analysis discussed above, we also used surveys and interviews. Statistics Norway helped us to distribute two surveys to the 
network of European NSIs. Both surveys had only three questions each, to keep them simple and to increase the response rate. The first survey was intended to get an idea about how the NSIs are preparing for the WAD, raise awareness about detected barriers with accessibility evaluation results, and to collect some input on how evaluation results can be shaped to enable the NSIs to understand them and to use them to repair the reported barriers. The second survey was designed to capture good DV examples and developments in the WAD preparations. The first survey, sent out by Statistics Norway in October 2017 to about 80 representatives from 39 NSIs in Europe, received a response from about 20 percent. The second survey, sent in April 2018 to the same group of respondents, had a lower response rate of 13 percent. One possible reason for the lower response rate may be a focus on the General Data Protection Regulation (GDPR) at this time. Interviews were carried out with Statistics Norway and with Eurostat.

The practical responsibility to make sure that DVs are accessible lies with software developers or with communications departments within the NSIs. Useful accessibility input has in several cases been obtained from colleagues with visual impairments. External consultants are sometimes contracted to audit overall websites. This is a costly operation and therefore not carried out regularly. Advanced and regular usability testing has been in place for a long time across a number of NSIs, to ensure that the statistics can be found and used. It seems that accessibility is an emerging topic to be included in regular testing activities for NSI online content.

Several different automatic tools are used by the NSIs to evaluate accessibility. Commonly used tools are Site Morse (see https://sitemorse.com/) and aXe Core (see http://deque.com/). One of the NSIs also reported that they intend to build a new tool. Such tools are helpful and cost-effective to operate, but not always straightforward to use. One important caveat is that these tools do not cover all conceivable tests.

From the first survey we found that respondents planned to pursue mainly two different approaches to improving their website and DV accessibility. They planned to invest in human resources which include staff training programmes and hiring accessibility expertise consultants, and facelifts or complete redesigns of their website.

Together with the survey we provided a benchmarking list similar to Table 7.1. The respondents were asked to comment on the results form. The checker tool (WTKollen, European NSI sites, 2017) was perceived as useful by respondents, and the findings verified that older or more complex webpages are more likely to have accessibility barriers than newer or simpler pages. For future tool development, respondents said that they would like to have a readability test and image evaluations. There was also a suggestion to group 
webpages according to complexity for better comparisons. To improve the presentation of the results in the checker report, the following suggestions were made:

- The results could have example images for better and faster understanding.

- The results could be sorted (e.g. by error status or importance)

- The page could be responsive

- It would be nice to be able to export all the errors to .csv/.pdf/.xlsx, as this would help the organization of corrections.

Some respondents raise concerns about tools since they do not always return the same result for the same content on a webpage. Such differences may even have prevented people from using checker tools at all. We also noted that some NSIs expected an official tool to be prescribed by the European Commission or their national ministry. However, the WAD is prepared in a way that is tool independent and there is no tool mentioned in the WAD implementation act.

In the second survey we requested users to provide examples of accessible DVs. Most respondents declined and indicated that they were working on this now. In our view, there is great potential in using DV templates from Eurostat to spread good accessible practices. We also asked about NSIs' preparations for the provision of accessibility statements and feedback mechanisms. Several NSIs have accessibility statements, sometimes linked from the page footer. However, in general, such statements do not list known deviations from accessibility requirements. Mostly they provide information about accessibility features of the sites. One NSI has had an accessibility statement since 2005 and regularly performs tests in cooperation with external experts. Many respondents aim to collect the information for the accessibility statement from the accessibility reports produced by officially adopted verification tools, or from user feedback.

In terms of preparations for the provision of feedback mechanisms, we recorded two approaches. One is to use a dedicated email to receive reports about accessibility problems. This approach can make it difficult for a user to remain anonymous, and it can also become hard to manage responses and task assignments for large volumes of feedback. The second approach is to use a general feedback mechanism already existing on the website. This may meet the formal requirements, but such feedback mechanisms are not designed to (automatically) collect data on accessibility problems, or to export reports about them to share good practices in terms of fixes or repair approaches. 


\section{Sharing good practices}

There are at least three current approaches to reusing good DV examples. The Digicom project is an initiative to share good practices among the NSIs. As part of this project, Eurostat has developed templates to present DVs of statistics. These templates are designed so that they are easy to translate and connect with an API to the data from Eurostat.

There are also a range of DV libraries that can be used to reuse good DV examples, like $\mathrm{D}_{3}$, Google charts, or Highcharts. In more accessible solutions, DVs are scalable to allow for zooming, and have functionality to encourage or force the developer to describe the non-textual elements. Such encouragement could raise developers' awareness of inaccessibility impacts for users with disabilities. A simple export of the data can also be helpful to enable users to explore the data with a tool of their own preference.

Presentation through aggregators like Google can also be efficient. The Google Public Data Explorer (see https://support.google.com/publicdata) provides large, public-interest datasets from sources like Eurostat and the World Bank in a common presentation format. With this service, the user can find datasets and explore them with different chart types. Two important advantages of this approach are, first, that users will be familiar with the user interface and, second, that they will easily find it, since Google has over 90 percent of the European search market share (StatCounter GlobalStats, 2018). However, such intermediary access can also be used to track users and to prevent the user from finding the original data source with more updated data or further information about the dataset.

We have not been able to identify a reference library of DVs. The Internet Archive is a valuable resource for longtime references for a large portion of the online content. Unfortunately, this archive does not have all the relevant pages from the NSIs and it cannot store the dynamic features of most dynamic DVs. The Internet Archive also will not have direct access to the static databases often serving the live' data to the dynamic DVs.

\section{Conclusions}

There are good examples of DVs where we found few accessibility barriers. However, despite the Web Accessibility Directive, there is still lot of room for improvement. There are several different accessibility testing tools in use among NSIs to test the accessibility of their websites and their DVs. In our survey we were not able to find examples of NSIs who systematically 
apply user testing approaches to uncover accessibility issues. Several report that they occasionally ask a colleague with disabilities to test content. For the WAD preparations, we saw very limited work towards design of an accessibility statement or to organize a feedback mechanism.

In general, NSIs are aware of accessibility issues. Still, three factors seem to have hindered focused progress towards comprehensive accessibility provisions, and to prepare for the WAD. Several NSIs indicated that they would wait for the WAD implementation act to be finalized before they would take action. The General Data Privacy Regulation seemed to demand more attention, as there are high fines associated with a breach compared to accessibility problems which breach the WAD. The third reason is differences in accessibility checker tool reports for the same element on a webpage. Some NSIs expect that an official tool will be named. The draft implementation act for the WAD does not refer to any named accessibility tool, and there seems to be no intention to use a particular tool for the implementation from the regulators as far as we have been able to find out.

There are several different accessibility testing tools in use among NSIs to test the accessibility of their websites and their DVs. For exploratory, interactive visualization we have found six different tools in use. Given this relatively small number of tools, targeted improvements of them can have a large effect for many users. Whatever approach is used, the central role of the NSIs and their DVs in national democratic discourse calls for particular awareness of accessibility.

\section{Acknowledgements}

To the INDVIL team for all the fruitful discussions and inputs, to the WTKollen project for providing the accessibility checker tools to test websites, webpages with DVs, and PDF documents. And last but not least, many thanks to Helen Kennedy for her helpful comments and substantial support to shape this chapter.

\section{References}

5-star data. (2015). 5 star open data. Retrieved October 22, 2018 from https://5stardata. info/en/

Coyne, K., \& Nielsen, J. (2001). Beyond ALT text:Making the web easy to use for users with disabilities. Fremont, CA: Nielsen Norman Group. 
EUR-Lex. (2005). Decision No 456/2005/EC of the European Parliament and of the Council of 9 March 2005 establishing a multiannual community programme to make digital content in Europe more accessible, usable and exploitable. Retrieved from https://eur-lex.europa.eu/legal-content/EN/TXT/?qid=1535296253785\&u $\mathrm{ri}=\mathrm{CELEX}: 32005 \mathrm{D} 0456$

Eurostat-Statistics Explained. (2017). Welcome to statistics explained. Retrieved October 22, 2018 from http://ec.europa.eu/eurostat/statistics-explained/index. php/Main_Page

GitHub. (2018). Axe Core tests. Retrieved October 22, 2018 from https://github.com/ dequelabs/axe-core/blob/master/doc/rule-descriptions.md

Google public data. (2018). Public data. Retrieved October 22, 2018 from https:// www.google.com/publicdata/explore

Jacob, E. K., \& Loehrlein, A. (2009). Information architecture. Annual Review of Information Science and Technology, 43(1). https://doi.org/10.1002/aris.2009.1440430110

Kennedy, H., Thomas, S., \& Evans, S. (2010) Can the web be accessible for people with intellectual disabilities? The Information Society, 27(1), 29-39. https://doi. org/10.1080/01972243.2011.534365

Kirk, A. (2012) Data visualization: A successful design process. Birmingham: Packt Publishing Ltd.

StatCounter GlobalStats. (2018). Search engine market share Europe. Retrieved October 22, 2018 from http://gs.statcounter.com/search-engine-market-share/ all/europe

$\mathrm{W}_{3}$ C. (2008). Web content accessibility guidelines (WCAG) 2.0. Retrieved October 22, 2018 from http://www.w3.org/TR/WCAG20/

W3C. (2014). Website accessibility conformance evaluation methodology (WCAGEM) 1.o. Retrieved October 22, 2018 from https://www.w3.org/TR/WCAG-EM/

W3C. (2016). Using longdesc. Retrieved October 22, 2018 from http://www.w3.org/ TR/WCAG2o-TECHS/H45.html

$\mathrm{W}_{3}$ C. (2018). Web content accessibility guidelines (WCAG) 2.1. Retrieved October 22, 2018 from https://www.w3.org/TR/WCAG21/

WTKollen-European NSI sites. (2017). Checked sites from European NSI sites. Retrieved October 22, 2018 from http://checkers.wtkollen.se/eu-nsi

WTKollen-European NSI sites. (2018). Checked sites from European NSI sites. Retrieved October 22, 2018 from http://checkers.wtkollen.se/en/benchmarking/testrunresults/d235468e-65a4-43b2-8428-59o8fo61fff9?Sector\&flags. fulltestrunresult

WTKollen-Page Checker. (2018). Check the accessibility of a web page. Retrieved October 22, 2018 from http://checkers.wtkollen.se/

Wikipedia, Findability. (2018). Findability. Retrieved October 22, 2018 from https:// en.wikipedia.org/wiki/Findability 


\section{About the authors}

Mikael Snaprud is the CEO of Tingtun AS. He has managed several projects in the area of e-Inclusion with European and national research grants. He has 20 years of experience from research and teaching, and has co-authored reports about ICT policies for the European Commission and the United Nations.

Andrea Velazquez works as scientific adviser at Tingtun AS. She has been part of the team of several e-Government projects funded by the European Commission and the Research Council of Norway. In the past she worked in processes benchmarking for quality engineering, and productivity programmes in the industry. 